\title{
Estudio comparativo de los enfoques de aprendizaje de los estudiantes de los Grados en Pedagogía y Educación Primaria
}

\section{Comparative study of the learning approaches of Pedagogy and Primary Education Bachelors' students}

\author{
Ana Belén Mirete Ruiz \\ anabelen.mirete@um.es \\ Raquel PÉrez Rubio \\ raquel.perez11@um.es \\ Javier J. Maquilón Sánchez \\ jjmaqui@um.es \\ Facultad de Educación. Universidad de Murcia, Murcia, España
}

\section{Resumen:}

El Espacio Europeo de Educación Superior permitió introducir profundos cambios en las Universidades a nivel estructural, académico, organizativo y de gestión, aunque para los estudiantes se materializaron en cambios relacionados con sus procesos de aprendizaje y los modos de abordar las demandas que la institución y el profesorado les realizó.

Una vez superado el horizonte del 2010 y con la mirada puesta en el horizonte 2020 , pero en pleno proceso de renovación de las acreditaciones de los títulos universitarios de grado y máster, surge la necesidad de reflexionar si los resultados obtenidos tras toda esta reforma han sido los esperados, o deberían acometerse nuevas reformas.

Desde esta perspectiva se presenta una investigación que analizó el perfil y los

\begin{abstract}
:
The European Higher Education Area allowed the introduction of significant changes in universities at the structural, academic, organizational and management levels, although for the students those changes were materialised in changes related to their learning processes and in the ways of dealing with the demands asked by the institution and professors.

Once the Horizon 2010 has passed and focusing on the Horizon 2020, while still being in the middle of the process of renovating the certification of bachelor and master titles, the necessity of reflecting on the results obtained after the whole reform comes in order to prove if those results are the ones expected or new reforms should be undertaken.

From this perspective, an investigation that analysed the profile and learning approa-
\end{abstract}

1 Dirección para correspondencia (correspondence address):

Ana Belén Mirete Ruiz. Dto. De Didáctica y Organización Escolar. Facultad de Educación. Facultad de Educación. Universidad de Murcia. Campus de Espinardo, s/n. 30100 Murcia (España). 
Estudio comparativo de los enfoques de aprendizaje de los estudiantes de los Grados en Pedagogía y Educación Primaria

Ana Belén Mirete Ruiz, Raquel Pérez Rubio y Javier J. Maquilón Sánchez

enfoques de aprendizaje de 416 estudiantes universitarios del grado de Educación Primaria y del Grado de Pedagogía, a lo largo de los tres primeros cursos.

El diseño de investigación seguido fue cuantitativo no experimental tipo encuesta. Para la recogida de información se empleó es Cuestionario de Proceso de Estudio en su versión revisada a dos factores (CPE-R-2F). Los datos fueron analizados con el paquete estadístico SPSS versión 23. Los resultados obtenidos apuntan a que el enfoque de aprendizaje más empleado por los estudiantes es el profundo y que además, su porcentaje de uso se incrementa curso a curso. Estos datos están relacionados con la calidad del aprendizaje y el rendimiento académico que, a priori, debería ser adecuado.

\section{Palabras clave:}

enfoques de aprendizaje; educación superior; educación primaria; pedagogía. ches of 416 students of the bachelors of Primary Education and Pedagogy during three academic years is presented.

The design followed in this investigation is a non-experimental quantitative study carried out through a survey. The Study Process Questionnaire-2 Factors (SPQ) in its revised and Spanish version (R-CPE$2 \mathrm{~F})$ has been used for the data collection. The results were analysed with the statistic package SPSS in its $23^{\text {rd }}$ version.

The results showed that the predominant learning approach is the deep approach and that its percentage of use increases with the courses. These results are related to the quality of learning and the academic performance, both being, a priori, adequate.

\section{Key words:}

learning approaches; higher education; primary education; pedagogy.

\section{Résumé :}

L'Espace Européen d'Enseignement Supérieure a permis introduire des profonds changements dans les Universités au niveau structurel, académique, organisationnel et de gestion. Pour les étudiants les changements se sont traduits par des modifications liées aux processus d'apprentissage et manières d'aborder les demandes de l'institution et du professorat.

Une fois que l'horizon de 2010 a été surpassé et avec le regard mis en 2020, mais en plein processus de renouvellement des accréditations des diplômes universitaires de degré et de master, le besoin s'en fait sentir de réfléchir si les résultats obtenus après toute cette réforme ont été appropriés ou doivent s'acquitter à nouveaux changement. De ce point de vue, on présente une recherche qui a analysé le profil et les approches d'apprentissage de 416 étudiants universitaires du degré d'enseignement primaire et du degré de pédagogie, sur une pèriode de trois premiers cours.

Le projet de recherche a été d'ordre quantitatif non expérimental pour sondage. Pour la collecte d'information s'est employé le Questionnaire de Processus d'Étude dans sa version révisée à deux facteurs (R-CPE-2F). Les données ont été analysées avec le paquet statistique SPSS version 23.

Les résultats obtenus indiquent que les étudiants emploient une approche d'apprentissage plus profond en augmentant son pourcentage d'usage cours à cours. Ces données sont liées à la qualité d'apprentissage et les résultats scolaires qui devrait être appropriés.

\section{Mots clés:}

approches d’apprentissage; enseignement supérieures; enseignement primaire; pédagogie.

Fecha de recepción: 15-5-2016

Fecha de aceptación: 23-6-2016 


\section{Introducción}

El interés por conocer las concepciones que profesorado y estudiantes tienen de lo que significa enseñar y aprender, y cómo ello afecta a los procesos educativos y a la mejora de la calidad de los mismos es un tema de suma relevancia. La preocupación por descubrir de qué manera los estudiantes se enfrentan al proceso de aprendizaje cuenta con numerosos estudios realizados en las últimas décadas, dando lugar a modelos que tratan de explicar cómo se produce dicho proceso (Biggs, 1989, Kember, 2000, Maquilón y Hernández, 2011, Maquilón, Mirete, García y Hernández, 2013, Maquilón, Sánchez y Cuesta, 2016, Mirete, 2014).

De forma paralela al inicio de las investigaciones centradas en las concepciones de aprendizaje, surgen los primeros trabajos que indagan sobre la manera en que los estudiantes conciben sus procesos de aprendizaje. Encontramos los orígenes de la investigación sobre las diferentes maneras de concebir el aprendizaje por parte de los estudiantes en los trabajos de Perry (1970, citado en Torre, 2007). En ellos se describe el planteamiento de los estudiantes desde una perspectiva de niveles de desarrollo epistemológico y sitúa las diferentes formas de razonamiento en un continuo que va desde un razonamiento concreto y dualista hasta otro más comprometido, con un nivel intermedio donde el razonamiento es contingente y relativista.

Perry (1970, citado en Torre, 2007) plantea una evolución en la manera de percibir el aprendizaje marcada por el tiempo de permanencia en la institución, lo cual hace que los estudiantes vayan comprendiendo que el aprendizaje es más gratificante cuando se dirige hacia la transformación de la información y la construcción de conocimiento (Torre, 2007). Desde este planteamiento surge el concepto de enfoques de aprendizaje (approaches to learning) que fue introducido por Marton y Säljö para referirse a la adaptación de estrategias de estudio que llevan a cabo los alumnos para afrontar distintas tareas a lo largo de su vida como estudiantes. Dicha definición fue el origen para un marco conceptual conocido como "la teoría de los enfoques de aprendizaje de los estudiantes" (Student Approaches to Learning, SAL)" (Biggs, Kember y Leung, 2001, p.134). Marton y Säljö (1976) fueron, los primeros estudiosos en emplear los términos enfoque profundo y superficial del aprendizaje. Dichos autores realizaron una investigación con estudiantes universitarios a partir de la lectura de un artículo, clasificando las estrategias seguidas para su 
comprensión según dichos términos. El enfoque superficial lo utilizaron al referirse a aquellos estudiantes que tenían una concepción reproductora del aprendizaje y el enfoque profundo lo utilizaron al referirse a los estudiantes que mostraban un mayor interés por el significado de los que estaban aprendiendo.

Entwistle (1981, citado en Torre, 2007) analizó las relaciones existentes entre los enfoques de aprendizaje y los niveles de comprensión o procesamiento. En la Tabla 1 se sintetiza su aportación.

\section{Tabla 1. Enfoques de aprendizaje y niveles de comprensión.}

\begin{tabular}{cl}
\hline Enfoque de aprendizaje & Nivel de procesamiento \\
\hline Profundo-Activo & $\begin{array}{l}\text { Comprende el significado del autor y demuestra la } \\
\text { evidencia que apoya la argumentación. }\end{array}$ \\
\hline Profundo-Pasivo & $\begin{array}{l}\text { Menciona el argumento principal, pero no vincula } \\
\text { la evidencia con la conclusión. }\end{array}$ \\
\hline Superficial-Activo & $\begin{array}{l}\text { Describe los puntos principales sin integrarlos den- } \\
\text { tro de un argumento. }\end{array}$ \\
\hline Superficial-Pasivo & Menciona unos pocos puntos aislados o ejemplos. \\
\hline Fuente: Entwistle (1981, citado en Torre, 2007, p.185).
\end{tabular}

La naturaleza contextual que influye en el proceso de aprendizaje de los estudiantes quedó reflejada por Biggs (1989; 1991) en el Modelo 3P de aprendizaje (Figura 1). Este modelo fue desarrollado originalmente por Dunkin y Biddle (1974, citado en Prosser \& Trigwell, 2006), y posteriormente redefinido por Biggs. En él se analiza el aprendizaje de los estudiantes desde una perspectiva fenomenológica y contextual, y "se hace eco de distintos sistemas anidados que son relevantes en el aprendizaje de los estudiantes: el sistema del estudiante, el sistema de la clase, el sistema institucional y el sistema comunitario" (Hernández, García \& Maquilón, 2004, p.99). El modelo formula el proceso de enseñanza y aprendizaje como un proceso interactivo, donde todos sus componentes se relacionan y potencian respectivamente. 


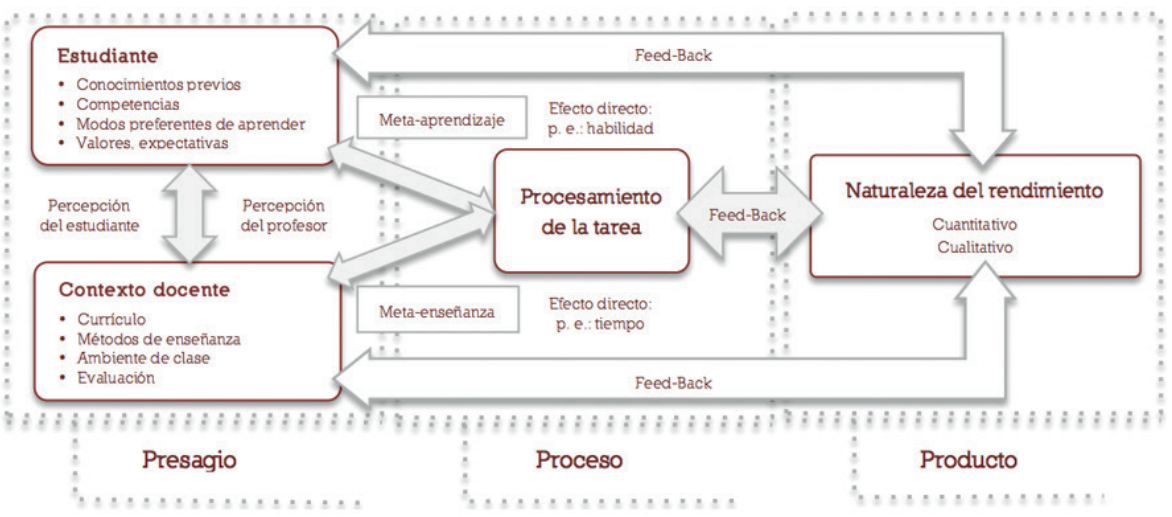

Figura 1. Modelo de aprendizaje 3P - Biggs (1989).

Los factores o variables de presagio comprenden los aspectos contextuales (del estudiante y de la enseñanza) previos a la acción educativa en un aula, aquí interactúan las características individuales de los estudiantes y sus competencias cognitivas con los conocimientos previos, motivación para el estudio, etc., y los aspectos relacionados con el contexto de la enseñanza, como la cultura educativa de la institución, los métodos de enseñanza que emplea el profesorado, los procesos de evaluación, su experiencia como docente, etc. Estas variables de presagio van a condicionar las variables de proceso, siendo este el momento en el que el estudiante selecciona y moviliza sus competencias en función del enfoque que adopte para resolver las tareas que se le demandan (Ramsden, 1988).

El enfoque de aprendizaje está condicionado por las estrategias que el estudiante sea capaz de emplear para superar sus situaciones de aprendizaje, pero sobre todo por la motivación que le guíe para hacerlo, ya que la motivación mueve a la conducta y permite provocar cambios no sólo a nivel educativo sino también en la propia vida. Ésta es una variable muy importante, ya que no existen ningún modelo de aprendizaje que no incorpore una teoría de motivación implícita o explícita. El estudiante siempre tiene una intención al realizar las tareas académicas según sean sus actitudes, percepciones y expectativas. Sus metas a alcanzar, constituirán factores que guían y dirigen su conducta en el ámbito académico. En paralelo, la motivación de una persona también se verá influida por los factores procedentes del contexto en el que se desenvuelve, ya sea económico, social, familiar, etc. Identificar y analizar 
estos factores ha sido objeto de múltiples investigaciones, tanto a nivel nacional como internacional (Abalde, Barca, Muñoz \& Ferrando, 2009, Buendía \& Olmedo, 2002, 2003, Corominas, Tesouro \& Teixidó, 2006, Entwistle \& Tait, 1990, Hernánez, García, Martínez, Hervás y Maquilón, 2002, Maquilón y Hernández, 2011, Maquilón et al., 2013, Maquilón et al., 2016, Mirete, 2014, Olmedo, 2013, Recio \& Cabero, 2005, Trigwell, Ellis, \& Feifei, 2011, Ramsdem, 1979).

Según Muñoz (2010), existen determinadas variables personales que influyen sobre la motivación académica y que deben ser tenidas en cuenta para mejorar la calidad de los aprendizajes de los estudiantes, para aumentar el rendimiento académico o para evitar el fracaso escolar:

- El autoconcepto. Considerado como el resultado de un proceso de análisis, valoración e integración de la información derivada de la propia experiencia y de la retroalimentación de los otros significativos como compañeros, padres y profesores. Se regula la conducta mediante un proceso de autoevaluación de modo que el comportamiento de un estudiante en un momento determinado, está influido en gran medida por su autoconcepto en ese instante.

- Los patrones de atribución causal. Que están determinados, en gran medida, por las consecuencias afectivo-emocionales derivadas de la realización de la tarea, así como de los éxitos y fracasos obtenidos de la misma.

- Las metas de aprendizaje. Definidas como los objetivos que pretende conseguir el estudiante. Condicionan los modos preferentes de afrontar las tareas académicas y distintos patrones motivacionales. Esto da lugar a que se consideren dos tipos de motivación, la motivación intrínseca y la motivación extrínseca.

La motivación intrínseca proviene del interior del sujeto. Se evidencia cuando el individuo realiza una actividad por el simple placer de realizarla, sin que nadie le incentive externamente. Un hobby es un ejemplo típico, así como la sensación de placer, la autosuperación o la sensación de éxito. Maslow (1943), en sus investigaciones de motivación, considera que si el ser humano satisface sus necesidades primarias (biológicas, seguridad y pertenencia) y las secundarias (reconocimiento y estima), Ilega finalmente a la autorrealización, aspecto que buscan las personas con mayor motivación intrínseca.

Según Maquilón (2001), la motivación intrínseca se genera en mu- 
chos individuos atraídos por la inquietud en conocer el mundo o explicar fenómenos. El éxito en la superación de los retos es uno de los ingredientes principales de la motivación intrínseca, con lo cual está muy ligada al enfoque profundo de aprendizaje.

En el otro extremo se encuentra la motivación extrínseca, definida como aquella que procede de fuera y que conduce a la ejecución de la tarea. Todas las clases de emociones relacionadas con resultados se asume que influyen en la motivación extrínseca de tareas.

El estudio de la motivación extrínseca se basa en los tres conceptos principales de recompensa, castigo e incentivo. Las recompensas y los castigos se dan después de la conducta y aumentan o reducen la probabilidad de que de vuelva a repetir, mientras que los incentivos se dan antes que la conducta y energizan su comienzo. Las personas extrínsecamente motivadas actúan para conseguir motivadores extrínsecos tales como dinero, elogios o reconocimiento social. Los dos tipos de motivación (intrínseca y extrínseca) son complementarios, y pueden ser combinados para producir un nivel máximo de motivación. De hecho, la motivación extrínseca puede ser útil para iniciar una actividad, pero ésta sólo puede ser mantenida mediante los motivadores intrínsecos pertinentes.

La conjunción adecuada de estrategias y motivaciones van asociadas a los resultados académicos, entendidos en términos de rendimiento. Dentro del modelo 3P de aprendizaje se relacionarían con las variables de producto (Biggs, 1989) y ese nivel de adecuación puede correlacionar con el éxito o el fracaso educativo, constituyendo un problema de extraordinaria importancia dentro del sistema educativo. La situación de fracaso puede provocar una serie de problemas y tensiones emocionales que repercuten en el desarrollo personal de los estudiantes o en su motivación hacia el abandono (Mena, Fernández-Enguita y Riviére, 2010), como son la repetición de curso (88\%) y el absentismo (91\%).

De Natale (1990) define el rendimiento académico como el conjunto de habilidades, destrezas, hábitos, ideales, aspiraciones, intereses, inquietudes y realizaciones que aplica el estudiante para aprender. La caracterización de los enfoques de aprendizaje de los estudiantes podría ser empleada para identificar aquellos estudiantes que se encuentran en una situación que oriente al fracaso escolar que según Calero, Choi y Waisgrais (2009) debe ser entendido como la proporción de individuos que no consiguen concluir los estudios obligatorios.

Desde esta perspectiva, el objetivo general de este estudio se centró 
en realizar un análisis comparativo de los enfoques de aprendizaje de los estudiantes del Grado en Educación Primaria y el Grado en Pedagogía. Para ello, se plantean los siguientes objetivos específicos: (1) Analizar los enfoques de aprendizaje de los estudiantes del Grado en Educación Primaria a nivel general y según el curso, (2) Analizar los enfoques de aprendizaje de los estudiantes del Grado en Pedagogía a nivel general y según el curso, y (3) Comparar los enfoques de aprendizaje de los estudiantes de los Grados en Pedagogía y Educación Primaria.

\section{Método}

\section{Participantes}

Se contó con la participación de 416 estudiantes de la Facultad de Educación, de los cuales 136 (32.7\%) estaban matriculados en el Grado en Pedagogía. Del Grado en Educación Primaria participaron 280 estudiantes $(67.3 \%)$. Su distribución según el curso se presenta en la Tabla 1.

Tabla 1. Distribución de los participantes según Grado y curso.

\begin{tabular}{lcccc} 
& $1^{\text {er }}$ Curso & $2^{\circ}$ Curso & $3^{\text {er }}$ Curso & Total \\
\hline Grado en Educación Primaria & 156 & 61 & 63 & 280 \\
\hline Grado en Pedagogía & 39 & 45 & 52 & 136 \\
\hline Total & 195 & 106 & 115 & 416 \\
\hline
\end{tabular}

\section{Instrumento}

Para la recogida de información en esta investigación se empleó el Cuestionario de Procesos de Estudio CPE-R-2F en su versión revisada a 2 factores (Biggs et al., 2001), traducido y adaptado al castellano por el equipo de la profesora Hernández-Pina (Hernández et al., 2002), y revisado por Hernández-Pina y Monroy en el año 2012 (Monroy, 2013), siendo ésta la versión empleada en la investigación. Este cuestionario analiza los enfoques de aprendizaje de estudiantes universitarios a partir de la percepción que tienen de sus propias motivaciones y estrategias de aprendizaje en el ámbito académico a nivel universitario.

El instrumento se compone de 20 ítems distribuidos en dos escalas (enfoque superficial y enfoque profundo) acompañados de una escala 
tipo Likert de cinco valores, siendo 1 el valor que representa una situación de aprendizaje que ocurre nunca o casi nunca, y 5 cuando ésta ocurre siempre o casi siempre.

\section{Diseño}

Para llevar a cabo la investigación se empleó un diseño cuantitativo no experimental tipo encuesta. Se seleccionó este diseño por ser un método de investigación capaz de dar respuesta a problemas tanto en términos descriptivos como de relación de variables (Hernández y Maquilón, 2010).

\section{Procedimiento}

El procedimiento llevado a cabo ha seguido las fases establecidas en el diseño survey o tipo encuesta. Inicialmente se seleccionó un instrumento adecuado a los objetivos de esta investigación, validado previamente en estudios previos (Hernández et al., 2004, Monroy, 2013, Mirete, 2014).

Previa solicitud al profesorado responsable de los grupos del tiempo necesario (aproximadamente 15 minutos) para la aplicación de los cuestionarios, se acudió en fecha y hora concertada a las diferentes clases. Antes de llevar a cabo dicha aplicación, se realizó una breve explicación del objeto de investigación, de la composición del cuestionario y el proceso de cumplimentación. Se garantizó el anonimato, se informó sobre la posibilidad de no participar. Todos los estudiantes cumplimentaron el cuestionario.

Los datos recogidos fueron depurados y analizados con el paquete estadístico SPSS V23 para MAC.

\section{Resultados}

Las publicaciones científicas sobre los enfoques de aprendizaje suelen iniciarse con un análisis de las puntuaciones obtenidas en las subescalas que componen el CPE-R-2F. En este trabajo, este análisis no ha sido incluido ya que, tanto en esta como en otras publicaciones (Gargallo, Garfella y Pérez, 2006; González, Del Rincón y Del Rincón, 2011; Kember, Biggs y Leung, 2004; Mirete, 2014), se ha constado que, si bien el CPE$\mathrm{R}-2 \mathrm{~F}$ es un instrumento válido y fiable para medir los enfoques de apren- 
dizaje, no debe ser empleado para identificar y analizar por separado las subescalas (motivos y estrategias), ya que los enfoques se establen en el plano de un continuo difícilmente categorizable.

En el primer objetivo se analizaron los enfoques de aprendizaje de los estudiantes del Grado en Educación Primaria a nivel general y según el curso.

El análisis de los enfoques de aprendizaje de los estudiantes del Grado en Educación Primaria la UM a nivel general (Figura 1), se constató la existencia de un mayor porcentaje de estudiantes con un enfoque profundo $(65.7 \% ; \mathrm{N}=184)$ que de estudiantes con un enfoque superficial $(30.7 \% ; \mathrm{N}=86)$. También se observa que un porcentaje de estudiantes $(3.6 \% ; \mathrm{N}=10)$ que tienen un perfil de aprendizaje disonante, debido a que obtuvieron idéntica puntuación en ambos enfoques.

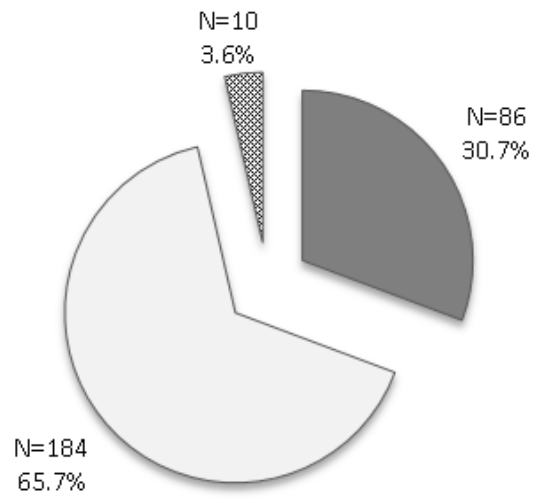

SA $\square$ DA 図 Disonante

Figura 1. Enfoque de aprendizaje a nivel general en Grado en Educación Primaria.

Los resultados obtenidos del análisis de los enfoques de aprendizaje (Tabla 2), mostraron que los estudiantes con un enfoque de aprendizaje predominantemente superficial obtuvieron una puntuación media de 29.14 puntos $(S d .=5.34)$, mientras que la puntuación obtenida por los aquellos alumnos con un enfoque profundo fue de 30.18 puntos $(\mathrm{Sd} .=5.44)$, indicando que los estudiantes que adoptan de forma predominante un enfoque profundo, lo hacen con mayor consistencia (motivos y estrategias propios del enfoque) que aquellos que adoptan un enfoque superficial. 
Tabla 2. Estadísticos de los enfoques de aprendizaje a nivel general en Grado en Educación Primaria.

\begin{tabular}{|c|c|c|c|c|c|c|}
\hline & \multirow{2}{*}{\multicolumn{2}{|c|}{$\begin{array}{l}\text { Estudio porcentual } \\
\text { por enfoque }\end{array}$}} & \multicolumn{4}{|c|}{$\begin{array}{l}\text { Puntuaciones medias de enfoque } \\
\text { por grupo }\end{array}$} \\
\hline & & & \multicolumn{2}{|c|}{ SA } & \multicolumn{2}{|c|}{ DA } \\
\hline & Frq. & $\%$ & M & Sd. & $M$ & Sd. \\
\hline Enfoque Superficial & 86 & 30.7 & 29.14 & 5.34 & 22.38 & 4.16 \\
\hline Enfoque Profundo & 184 & 65.7 & 20.67 & 4.42 & 30.18 & 5.44 \\
\hline Disonante & 10 & 3.6 & & & & \\
\hline Total & 280 & 100 & & & & \\
\hline
\end{tabular}

Cuando se analizó el rango existente entre las puntuaciones medias obtenidas dentro de cada enfoque en las dos escalas (SA y DA), se encontró que dicha distancia es mayor en el caso de los alumnos con un enfoque profundo (9.51 puntos), que aquellos que adoptan un enfoque superficial (distancia de 6.76 puntos). Estos datos evidencian que los alumnos que adoptan de manera predominante un enfoque profundo lo hacen de forma más consistente, haciendo un mayor uso de los motivos y estrategias propias del enfoque, alejándose de aquellas inherentes al enfoque superficial.

$\mathrm{Al}$ analizar el enfoque de aprendizaje según el curso en el que se encuentran matriculados los estudiantes del Grado en Educación Primaria. Tal y como se observa (Figura 2), el mayor porcentaje de estudiantes con un enfoque de aprendizaje superficial se encontró en los grupos de alumnos de primer curso ( $34.6 \%$; $\mathrm{N}=54)$ y segundo curso $(24.6 \% ; \mathrm{N}=15)$, mientras que el grupo que tienen un mayor porcentaje de alumnos que adoptan un enfoque profundo son los de tercer año $(73 \% ; \mathrm{N}=46)$. Finalmente, el grupo de estudiantes de segundo curso $(8.2 \% ; \mathrm{N}=5)$ es el que tiene el mayor porcentaje de estudiantes con un enfoque de aprendizaje disonante, mientras que no se encuentran estudiantes con dicho enfoque en el grupo de tercer curso.
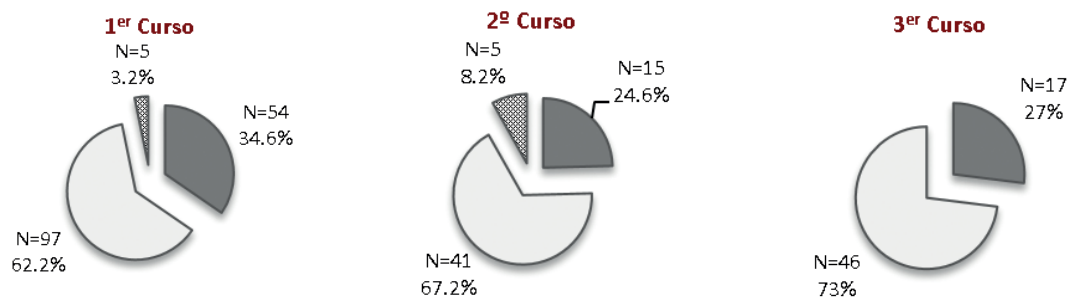

SA $\square$ DA 圆 Disonante

Figura 2. Enfoques de aprendizaje Grado en Educación Primaria según curso. 
El resultado del análisis de las puntuaciones medias obtenidas por los tres grupos de estudiantes se presenta en la Tabla 3. En el enfoque de aprendizaje superficial, los estudiantes de segundo curso son los que obtuvieron unos valores medios más elevados $(M=29.80$; $S d .=4.28)$, frente a los de primer curso que obtuvieron las puntuaciones medias más bajas $(M=28.98 ;$ Sd. $=5.41)$, aunque con una escasa diferencia. En el enfoque profundo se encontró que los alumnos de segundo año son los que puntúan más alto $(M=30.66$; $\mathrm{Sd}$. $=5.63)$, seguidos muy de cerca por los estudiantes de tercer curso $(M=30.39 ; \mathrm{Sd} .=5.43)$ y finalmente los alumnos de primer curso $(M=29.88 ; S d .=5.39)$.

Tabla 3. Estadísticos de los enfoques de aprendizaje en Grado en Educación Primaria según curso.

\begin{tabular}{|c|c|c|c|c|c|c|c|}
\hline & \multirow{3}{*}{$\begin{array}{c}\text { Enfoque } \\
\text { predominante }\end{array}$} & \multirow{2}{*}{\multicolumn{2}{|c|}{$\begin{array}{l}\text { Estudio por- } \\
\text { centual por } \\
\text { enfoque }\end{array}$}} & \multicolumn{4}{|c|}{$\begin{array}{l}\text { Puntuaciones medias de } \\
\text { enfoque por grupo }\end{array}$} \\
\hline & & & & \multicolumn{2}{|c|}{ SA } & \multicolumn{2}{|c|}{ DA } \\
\hline & & Frq. & $\%$ & M & Sd. & M & Sd. \\
\hline \multirow{4}{*}{$\begin{array}{l}1 \text { er } \\
\text { curso }\end{array}$} & Enfoque Superficial & 54 & 34.6 & 28.98 & 5.41 & 22.61 & 4.14 \\
\hline & Enfoque Profundo & 97 & 62.2 & 20.77 & 4.59 & 29.88 & 5.39 \\
\hline & Disonante & 5 & 3.2 & & & & \\
\hline & Total & 156 & 100 & & & & \\
\hline \multirow{4}{*}{$\begin{array}{l}2^{\circ} \\
\text { curso }\end{array}$} & Enfoque Superficial & 15 & 24.6 & 29.80 & 4.28 & 21.60 & 4.67 \\
\hline & Enfoque Profundo & 41 & 67.2 & 20.32 & 4.80 & 30.66 & 5.63 \\
\hline & Disonante & 5 & 8.2 & & & & \\
\hline & Total & 61 & 100 & & & & \\
\hline \multirow{4}{*}{$\begin{array}{l}3 \text { er } \\
\text { curso }\end{array}$} & Enfoque Superficial & 17 & 27.0 & 29.06 & 6.18 & 22.35 & 3.92 \\
\hline & Enfoque Profundo & 46 & 73.0 & 20.76 & 3.82 & 30.39 & 5.43 \\
\hline & Disonante & - & - & & & & \\
\hline & Total & 63 & 100 & & & & \\
\hline
\end{tabular}

En el análisis de la distancia entre las puntuaciones medias obtenidas dentro del enfoque superficial, se observan valores entre SA y DA que oscilan de 6.37 puntos (primer curso) y 8.20 puntos (segundo curso). Este mismo análisis de los estudiantes con un enfoque profundo, muestra una diferencia entre DA y SA que van de los 9.63 puntos en el grupo de estudiantes de tercero y los 10.34 puntos en el grupo de alumnos de segundo curso. Dichos resultados indican que los estudiantes que adoptan de forma predominante un enfoque profundo, lo hacen con un mayor afianzamiento que aquellos que adoptan un enfoque superficial. 
En el segundo objetivo se analizaron los enfoques de aprendizaje de los estudiantes del Grado en Pedagogía a nivel general y según el curso en el que se encuentran matriculados. Tal y como se observa en la Figura 3 , el $74.2 \%$ de los estudiantes del Grado en Pedagogía (74.2\%; N=101), adoptan de manera predominante un enfoque de aprendizaje profundo, frente al $22.1 \%(\mathrm{~N}=30)$ que lo hacen de forma superficial. Igualmente, se encuentra un porcentaje de alumnos $(3.7 \% ; \mathrm{N}=5)$ que adoptan un enfoque disonante (igual puntuaciones en ambos enfoques de aprendizaje).

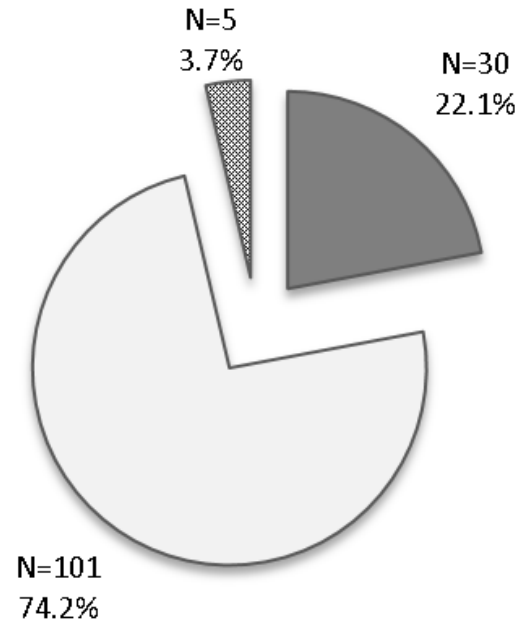

Figura 3. Enfoque de aprendizaje a nivel general en Grado en Pedagogía.

Seguidamente se presentan los resultados obtenidos del análisis de los enfoques de aprendizaje de los estudiantes del Grado en Pedagogía. Se puede observar (Tabla 4), que los estudiantes que adoptan predominantemente un enfoque superficial obtienen una puntuación media de 32.38 puntos $(\mathrm{Sd} .=6.02)$, mientras que la puntuación obtenida por los aquellos alumnos con un enfoque profundo es de 31.62 puntos $(\mathrm{Sd} .=5.02)$. Estos resultados indican que, los alumnos del Grado en Pedagogía que adoptan de forma predominante un enfoque superficial lo hacen más consistentemente que aquellos con un enfoque de aprendizaje profundo. 
Tabla 4. Estadísticos de los enfoques de aprendizaje a nivel general en Grado en Pedagogía.

\begin{tabular}{|c|c|c|c|c|c|c|}
\hline \multirow{3}{*}{$\begin{array}{c}\text { Enfoque } \\
\text { predominante }\end{array}$} & \multirow{2}{*}{\multicolumn{2}{|c|}{$\begin{array}{l}\text { Estudio } \\
\text { porcentual por } \\
\text { enfoque }\end{array}$}} & \multicolumn{4}{|c|}{$\begin{array}{l}\text { Puntuaciones medias de enfoque } \\
\text { por grupo }\end{array}$} \\
\hline & & & \multicolumn{2}{|c|}{ SA } & \multicolumn{2}{|c|}{ DA } \\
\hline & Frq. & $\%$ & M & Sd. & M & Sd. \\
\hline Enfoque Superficial & 30 & 22.1 & 32.38 & 6.02 & 24.72 & 5.29 \\
\hline Enfoque Profundo & 101 & 74.2 & 19.63 & 4.81 & 31.62 & 5.02 \\
\hline Disonante & 5 & 3.7 & & & & \\
\hline Total & 136 & 100 & & & & \\
\hline
\end{tabular}

Al analizar la distancia entre las puntuaciones medias obtenidas para cada uno de los enfoques en ambas escalas, encontramos que esta distancia es menor para el grupo de alumnos que adoptan un enfoque superficial (7.66 puntos), que para aquellos con un enfoque profundo (11.99 puntos). Estos resultados indican que los alumnos con un enfoque de aprendizaje profundo adoptan este enfoque de una manera más consistente que los que tienen un enfoque de aprendizaje superficial.

En la Figura 4 se presentan los resultados obtenidos del análisis del enfoque de aprendizaje de los estudiantes del Grado en Pedagogía según el curso en el que cursan sus estudios. Se observa que en el primer curso del grado existe un mayor porcentaje de estudiantes con un enfoque superficial ( $41 \% ; \mathrm{N}=16)$, seguido por los de segundo curso $(22.2 \%$; $\mathrm{N}=10$ ). En lo referente al enfoque de aprendizaje profundo, encontramos un mayor porcentaje de alumnos que adoptan este enfoque en tercer curso ( $88.5 \% ; \mathrm{N}=46)$. Nuevamente se encuentran casos de alumnos con un enfoque de aprendizaje disonante o no definido, siendo mayor el porcentaje de alumnos con este enfoque en primer año de Grado (5.2\%; $\mathrm{N}=2)$ y en tercero $(3.8 \% ; \mathrm{N}=2)$.
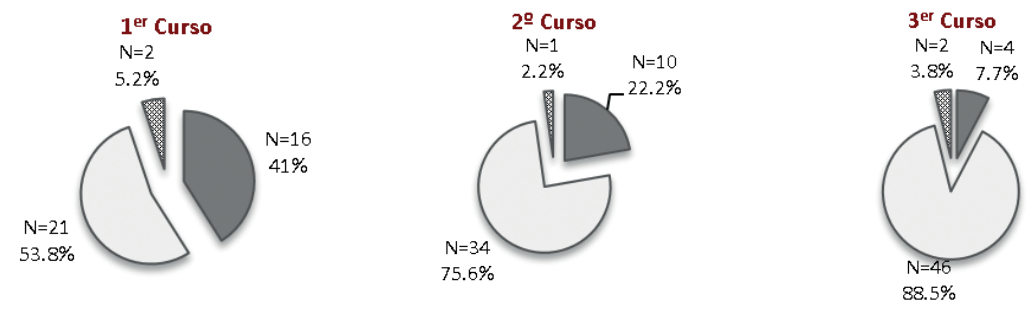

$\square \mathrm{SA} \square \mathrm{DA}$ Disonante

Figura 4. Enfoques de aprendizaje Grado en Pedagogía según curso. 
En la Tabla 5 se presentan los resultados obtenidos por los estudiantes en las subescalas que conforman los enfoques de aprendizaje según el curso de referencial Tal y como se puede observar, en el enfoque de aprendizaje superficial, son los alumnos de tercer curso los que obtienen unas puntuaciones medias más elevadas $(M=36.90 ; \mathrm{Sd} .=5.45)$, frente a los de primer curso que son los que obtienen las puntuaciones medias más bajas $(M=29.88 ; \mathrm{Sd} .=4.94)$. En el caso del enfoque profundo, al igual que ocurría en el enfoque superficial, son los alumnos de tercer curso los que puntúan más alto $(M=32.50 ; S d .=5.00)$, con valores medios próximos a los obtenidos por los estudiantes de segundo año $(M=32.38 ; \mathrm{Sd}$.=5.06).

Tabla 5. Estadísticos de los enfoques de aprendizaje en Grado en Pedagogía según curso.

\begin{tabular}{|c|c|c|c|c|c|c|c|}
\hline & \multirow{3}{*}{$\begin{array}{c}\text { Enfoque } \\
\text { predominante }\end{array}$} & \multirow{2}{*}{\multicolumn{2}{|c|}{$\begin{array}{l}\text { Estudio } \\
\text { porcentual por } \\
\text { enfoque }\end{array}$}} & \multicolumn{4}{|c|}{$\begin{array}{c}\text { Puntuaciones medias de } \\
\text { enfoque por grupo }\end{array}$} \\
\hline & & & & \multicolumn{2}{|c|}{ SA } & \multicolumn{2}{|c|}{ DA } \\
\hline & & Frq. & $\%$ & M & Sd. & M & Sd. \\
\hline \multirow{4}{*}{1 er curso } & Enfoque Superficial & 16 & 41.0 & 29.88 & 4.94 & 22.81 & 3.67 \\
\hline & Enfoque Profundo & 21 & 53.8 & 20.76 & 3.03 & 28.48 & 3.83 \\
\hline & Disonante & 2 & 5.2 & & & & \\
\hline & Total & 39 & 100 & & & & \\
\hline \multirow{4}{*}{$2^{\circ}$ curso } & Enfoque Superficial & 9 & 22.2 & 35.00 & 6.42 & 28.89 & 5.92 \\
\hline & Enfoque Profundo & 34 & 75.6 & 19.24 & 5.04 & 32.38 & 5.06 \\
\hline & Disonante & 1 & 2.2 & & & & \\
\hline & Total & 44 & 100 & & & & \\
\hline \multirow{4}{*}{ 3er curso } & Enfoque Superficial & 4 & 7.7 & 36.50 & 5.45 & 23.00 & 4.97 \\
\hline & Enfoque Profundo & 46 & 85.5 & 19.41 & 5.28 & 32.50 & 5.00 \\
\hline & Disonante & 2 & 3.8 & & & & \\
\hline & Total & 52 & 100 & & & & \\
\hline
\end{tabular}

Al analizar la diferencia encontrada entre las puntuaciones medias obtenidas dentro del enfoque superficial, se observó que los valores entre SA y DA oscilaron entre 6.11 puntos (segundo curso) y 13.50 puntos (tercer curso). En el caso del enfoque profundo, dichas las diferencias habidas entre DA y SA, van de los 7.72 puntos en el grupo de primero a los 13.14 puntos en el grupo de estudiantes de segundo. Los resultados encontrados muestran que, en el caso del Grado en Pedagogía, los alumnos adoptan con igual consistencia ambos enfoques. 
A continuación se da respuesta al tercer objetivo de esta investigación, el cual se centró en comparar los enfoques de aprendizaje de los estudiantes de los dos grados analizados. En la tabla 6 se incluyen las puntuaciones medias obtenidas por los estudiantes según el enfoque de aprendizaje predominante en cada grado. Se constata, tanto en el caso del enfoque superficial, como en el del enfoque de aprendizaje profundo, que los alumnos del Grado en Pedagogía obtuvieron puntuaciones medias superiores a las de los estudiantes del Grado en Educación Primaria.

Tabla 6. Puntuaciones medias de los enfoques de aprendizaje predominantes según el Grado cursado.

\begin{tabular}{|c|c|c|c|c|}
\hline \multirow{2}{*}{ Enfoque predominante } & \multicolumn{2}{|c|}{ Grado en Educación Primaria } & \multicolumn{2}{|c|}{ Grado en Pedagogía } \\
\hline & $M$ & $\mathrm{Sd}$ & M & Sd. \\
\hline Enfoque Superficial & 29.14 & 5.34 & 32.38 & 6.02 \\
\hline Enfoque Profundo & 30.18 & 5.44 & 31.62 & 5.02 \\
\hline
\end{tabular}

Una vez seleccionados los estudiantes que adoptan de forma predominante un enfoque de aprendizaje superficial, se ha realizado la prueba t de Student para muestras relacionadas para comparar las puntuaciones medias obtenidas por los alumnos de cada uno de los Grados. Los resultados obtenidos muestran que dichas diferencias son estadísticamente significativas $(t=2538.32 ; \mathrm{p}<.001)$. Del mismo modo, y seleccionados los estudiantes con un enfoque de aprendizaje profundo, la prueba $t$ $(t=4275.94 ; \mathrm{p}<.001)$ ha permitido comprobar que las diferencias halladas entre las medias obtenidas por los estudiantes del Grado en Pedagogía son estadísticamente superiores a las obtenidas por los del Grado en Educación Primaria.

\section{Discusión y conclusiones}

Una de las líneas clásicas de la investigación educativa está dirigida hacia la búsqueda de las características y factores que producen bajo rendimiento en los escolares y la prevención del fracaso escolar. La finalidad es clara, desarrollar estrategias, motivaciones y metodologías docentes innovadoras que puedan minimizar los efectos negativos de la falta de estrategias o la inadecuada motivación hacia las tareas académicas. 
En la investigación que se ha presentado se abordó esta problemática. Con los resultados obtenidos se puede afirmar que, en el grado de Educación Primaria, los estudiantes emplean mayoritariamente enfoque profundo de aprendizaje frente al enfoque superficial. Dos de cada tres estudiantes emplean un enfoque adecuado a la demanda de la institución, lo cual debe redundar en la calidad de los aprendizajes y en el rendimiento académico de estos estudiantes.

El enfoque profundo, en términos de media, es más consistente que el superficial, lo cual implica una elevada relación entre el uso de unas determinadas estrategias asociadas al enfoque y su motivación. En estudios previos se ha constatado los mismos resultados (Hernández et al., 2002, Hernández et al., 2004, Maquilón et al., 2011, Maquilón et al., 2013, Maquilón et al., 2016, Monroy, 2013, Mirete, 2014) aunque existían diferencias en cuanto al porcentaje de estudiantes que empleaban prioritariamente el enfoque profundo, ya que el más empleado en los estudiantes de este título (Hernández et al., 2002) era el superficial.

Resulta interesante la evolución del porcentaje de uso del enfoque superficial. Su mayor presencia se produce en primer curso, decreciendo en cursos posteriores. Es decir, que conforme los estudiantes van aumentando sus competencias en el título van mejorando su enfoque, pasando del superficial que habían importado de sus estudios previos hacia enfoques más profundos. Esta evolución hace que, a nivel global, tres de cada cuatro estudiantes de los grados analizados afirmen emplear predominantemente enfoque profundo en tercer curso.

También es necesario destacar que, a nivel global, en segundo curso existe casi un diez por ciento de estudiantes de Educación Primaria con enfoque disonante. Estudiantes que con un mínimo apoyo y asesoramiento pueden decantarse por el enfoque profundo frente al superficial. Enseñándoles estrategias adecuadas, mejorando su motivación hacia el aprendizaje, o indicándoles que aspectos de su motivación o estrategias superficiales están "lastrándoles", obtendríamos estudiantes cuyos resultados podrían ser mejores. Esto es un aspecto que debería plantearse a nivel del propio título ya que son muchos los estudiantes que cursan este grado.

Para los estudiantes con un enfoque predominantemente superficial del Grado en Educación Primaria y en términos de media, se encontró que no existen diferencias por curso. En cambio, los estudiantes con un enfoque predominantemente profundo se observaron que está más afianzado y se 
emplea de un modo más consistente que el superficial, además su uso consistente y coherente se incrementa progresivamente curso a curso. Es decir, que su motivación y sus estrategias de aprendizaje mejoran.

En el Grado en Pedagogía destacamos que, a nivel global, tres de cada cuatro estudiantes afirma emplear un enfoque predominantemente profundo. Estos datos son similares a los hallados en estudios previos (Hernández et al., 2002, Hernández et al., 2004).

En términos de media, el enfoque superficial está más afianzado que el profundo coincidiendo con las investigaciones de (Maquilón et al., 2013, Maquilón et al., 2016), lo cual indica que los estudiantes que predominantemente emplean un enfoque superficial (con las motivaciones y estrategias que lleva asociadas) lo hace de un modo más consistente. Este aspecto puede producir que difícilmente estos estudiantes puedan abandonar el enfoque superficial para pasar a enfoques más profundos. Máxime cuando en términos de media, el enfoque superficial está más afianzado curso a curso.

Consideramos muy positiva la evolución encontrada en el Grado en Pedagogía a nivel de curso, ya que, en primero, apenas uno de cada dos estudiantes empleaba enfoque profundo, aumentando progresivamente esta proporción, llegando a ser de cuatro de cada cinco en tercer curso. Obviamente, el porcentaje de estudiantes con un enfoque predominantemente superficial se ha ido reduciendo hasta ser inferior a un ocho por ciento. Este proceso de construcción personal, y de valor añadido por parte del título, resulta muy interesante y alentador, ya que este título tradicionalmente ha tenido una presencia mayoritaria de estudiantes con un enfoque superficial (Hernández et al., 2002).

Cuando se analizó el grado de afianzamiento de los enfoques (uso de estrategias de aprendizaje específicas de un enfoque relacionadas con la motivación específica del mismo) en base a los enfoques predominantes de cada Grado, se obtuvo que tanto en el caso del enfoque superficial, como en el del enfoque profundo, los alumnos del Grado en Pedagogía obtuvieron puntuaciones medias superiores a las de los estudiantes del Grado en Educación Primaria.

Al comparar las medias de los estudiantes que emplean predominantemente enfoque superficial mediante la prueba $t$ de Student se confirmó la existencia de diferencias estadísticamente significativas para los estudiantes del Grado en Pedagogía y los estudiantes del Grado en Educación Primaria. 
Igualmente, se seleccionó a los estudiantes con un enfoque predominantemente profundo, se aplicó la prueba $t$ de Student para muestras relacionadas y se comprobó que además de existir diferencias estadísticamente significativas, éstas son superiores para los estudiantes del Grado en Pedagogía frente a las obtenidas por los estudiantes del Grado en Educación Primaria.

Estos resultados vienen a confirmar que los cambios estructurales, académicos, organizativos y de gestión, que se acometieron en ambos títulos están teniendo unos resultados interesantes y alentadores para los estudiantes, sobre todo para los del Grado en Pedagogía, que tradicionalmente han sido estudiantes poco motivados y faltos de estrategias para superar las demandas de la institución y con una elevada tasa de abandono (Mena et al., 2010). Somos conscientes que este estudio, como la mayoría de los realizados en esta línea de investigación tiene sus carencias, pero resulta pertinente que en este proceso de renovación de la acreditación de los títulos pueda evidenciarse, con datos empíricos, que la calidad del aprendizaje de los estudiantes de los Grados en Educación Primaria y de Pedagogía evoluciona positivamente y que la institución a través del profesorado está potenciando una docencia y un aprendizaje de calidad.

\section{Referencias}

Abalde, E., Barca, A., Muñoz, J. M., y Ferrando, M. (2009). Rendimiento académico y enfoques de aprendizaje: una aproximación a la realidad de la enseñanza superior brasileña en la región norte. Revista de Investigación Educativa, 27(2), 303-319.

Biggs, J.B. (1989). Approaches to the enhancement of tertiary teaching. Higher Education Research and Development, 8(1), 7-25.

Biggs, J.B. (1991). Approaches to learning in secondary and tertiary students in Hong Kong: Some comparative studies. Educational Research Journal, 6, 27-39.

Biggs, J. B., Kember, D. y Leung, D. Y. P. (2001). The revised two-factor Study Process Questionnaire: R-SPQ-2F. British Journal of Educational Psychology, 71, 133-149.

Buendía, L. y Olmedo, E. M. (2002). El género: ¿Constructo mediador en los enfoques de aprendizaje universitario?. Revista de Investigación Educativa, 20(2), 511-524.

Buendía, L. y Olmedo, E. M. (2003). Estudio transcultural de los enfoques de aprendizaje en Educación Superior. Revista de Investigación Educativa, 21(2), 371-386.

Calero, J., Choi, A. y Waisgrais, S. (2009). Determinantes del riesgo de fracaso escolar en España: una aproximación a través de un análisis logístico multinivel aplicado a PISA-2006. Revista de Educación, Número Extraordinario 2010, 225-256. 
Estudio comparativo de los enfoques de aprendizaje de los estudiantes de los Grados en Pedagogía y Educación Primaria

Ana Belén Mirete Ruiz, Raquel Pérez Rubio y Javier J. Maquilón Sánchez

Campillo, S. (2005). Análisis del fracaso escolar en Beniel. Murcia: Azarbe.

Corominas, E., Tesouro, M. y Teixidó, J. (2006). Vinculación de los enfoques de aprendizaje con los intereses profesionales y los rasgos de personalidad. Aportaciones a la innovación del proceso de enseñanza y aprendizaje en la Educación Superior. Revista de Investigación Educativa, 24(2), 443-473.

De Natale, M. (1990). Rendimiento escolar. Diccionario de Ciencias de la educación. Argentina: Troquel.

Entwistle, N. J. y Tait, H. (1990). Approaches to learning, evaluations of teaching and preferences for contrasting academic environments. Higher Education, 19(2), 169-194.

Kember, D. (2000). Misconceptions about the learning approaches, motivation and study practices of Asian students. Higher Education, 40, 99-121.

Gargallo, B., Garfella, P. R. y Pérez, C. (2006). Enfoques de aprendizaje y rendimiento académico en estudiantes universitarios. Bordón, 58(3), 327-343.

González, J. L., Del Rincón, B. y Del Rincón, D.A. (2011). Estructura latente y consistencia interna del R-SPQ-2F: Reinterpretando los enfoques de aprendizaje en el EEES. Revista de Investigación Educativa, 29(2), 277-293.

Hernández, F. y Maquilón, J. J. (2010). Introducción a los diseños de investigación educativa. En S. Nieto (Ed.). Principios, métodos y técnicas esenciales para la investigación educativa. (pp.109-126). Madrid: Dykinson.

Hernández, F., García, M. P., Martínez, P., Hervás, R. y Maquilón, J.J. (2002). Consistencia entre motivos y estrategias de aprendizaje en estudiantes universitarios. Revista de Investigación Educativa, 20(2), 487-510.

Hernández, F., García, M. P. y Maquilón, J. J. (2004). Análisis del cuestionario de procesos de estudio-2 factores de Biggs en estudiantes universitarios españoles. Revista Fuentes, 6, 96-114.

Kember, D., Biggs, J. y Leung, D. Y. P. (2004). Examining the multidimensionality of approaches to learning through the development of a revised version of the Learning Process Questionnaire. British Journal of Educational Psychology, 74, 261-280.

Marton, F. y Säljö, R. (1976). Learning process and strategies. British Journal of Educational Psychology, 46, pp. 115-127.

Maslow, A. H. (1943). A theory of human motivation. New york: Pshychological Review.

Maquilón, J. (2001). Los enfoques de aprendizaje en estudiantes de Pedagogía. (Tesis de Licenciatura). Murcia: Universidad de Murcia.

Maquilón, J. J. y Hernández, F. (2011). Identificación de las características del aprendizaje de los estudiantes de Educación Primaria con el cuestionario CEAPS. Anales de psicología, 27(1), 126-134.

Maquilón, J. J., Mirete, A., García, F., y Hernández, F. (2013). Valoración de las TIC por los estudiantes universitarios y su relación con los enfoques de aprendizaje. Revista de Investigación Educativa, 31(2), 537-554.

Maquilón, J. J., Sánchez, M. y Cuesta, J. D. (2016). Enseñar y aprender en las aulas de Educación Primaria. Revista Electrónica de Investigación Educativa, 18(2), 144-155. Recuperado de http://redie.uabc.mx/redie/article/view/955

Mirete, A. B. (2014). TIC y enfoques de enseñanza y aprendizaje en Educación Superior. (Tesis Doctoral). Murcia: Universidad de Murcia. 
Estudio comparativo de los enfoques de aprendizaje de los estudiantes de los Grados en Pedagogía y Educación Primaria

ana Belén Mirete Ruiz, Raquel Pérez Rubio y Javier J. Maquilón Sánchez

Mena, L., Fernández-Enguita, M., Riviére, J. (2010). Desenganchados de la educación: procesos, experiencias, motivaciones y estrategias del abandono y del fracaso escolar. Revista de Educación. Número extraordinario, 119-145.

Monroy, F. (2013). Enfoques de Enseñanza y de Aprendizaje de los estudiantes del Máster Universitario en Formación del Profesorado de Educación Secundaria. (Tesis Doctoral). Murcia: Universidad de Murcia.

Muñoz, A. (2010). Identificación de los enfoques de aprendizaje empleados por los estudiantes de los Ciclos de Formación Profesional: Grado Medio vs Superior. Trabajo Fin de Máster. Universidad de Murcia.

Olmedo, E. M. (2013). Enfoques de aprendizaje de los estudiantes y metodología docente: Evolución hacia el nuevo sistema de formación e interacción propuesta en el EEES. Revista de Investigación Educativa, 31(2), 411-429.

Prosser, M. y Trigwell, K. (2006). Confirmatory factor analysis of the Approaches to Teaching Inventory. British Journal of Education Psychology, 76, 405-419.

Ramsden, P. (1979). Student learning and perceptions of the academic environment. Higher Education, 8, 411-427.

Ramsden, P. (1988). Improving learning; New perspectives. London: Kogan Page.

Recio, M.A. y Cabero, J. (2005). Enfoques de aprendizaje, rendimiento académico y satisfacción de los estudiantes en formación en entornos virtuales. Pixel-Bit. Revista de Medios y Educación, 25, 93-115.

Torre, J.C. (2007). Una triple alianza para un aprendizaje universitario de calidad. Madrid: Universidad Pontificia Comillas.

Trigwell, K., Ellis, R.A. y Feifei, H. (2011). Relations between students' approaches to learning, experienced emotions and outcomes of learning. Studies in Higher Education, 1-14. 
